\title{
The polio of our time: lyme disease
}

\author{
Volume 7 Issue I - 2017 \\ Katina Makris \\ Natural health care practitioner and educator, USA
}

\section{Opinion}

Lyme disease is a bacterial infection caused by an organism known as Borrelia burgdorferi. There are also variant tick-borne co-infections caused by similar bacteria, parasites, mycoplasmas and viruses. Rocky Mountain spotted fever, ehrlichia, babesia, and bartonella are related to lyme disease and all cause a similar set of symptoms with some minor yet detectable clinical characteristics differentiating them. Borrelia $B$ is classified as spirochete, or corkscrew bacteria, which is in the same family as syphilis, yet Lyme is considered to be stronger and more virulent, with more than 300 worldwide strains. Like syphilis, Lyme initially starts out with seemingly mild symptomology, but over time, if untreated, it can cause devastating effects to the central nervous system, heart, kidneys, skeletal and immune system with sometimes permanent repercussions.

Early diagnosis and detection of Lyme disease is critical. The sooner this bacterium is arrested, the more hopeful the prognosis is for a complete recovery. Do not hesitate to seek professional testing if you are at all suspicious you may have been infected. Original assumption was 2 weeks of oral doxycycline antibiotic would arrest the infection. The International Lyme and Associated Disease Society (ILADS) and researchers have discovered Borrelia $b$. takes 6weeks to complete its replication cycle unlike streptococcus or E. coli with 48hour replications. This means 6-8weeks of antibiotics are needed for even early Lyme infections.

The bacterium is spread by a blood transfer, most commonly transmitted by the bite of a very tiny insect- a tick. The small deer ticks are the size of a pinhead or small freckle. The larger dog tick and other tick varieties, found nationwide, also carry these organisms. Essentially, ticks are small 'cesspools' carrying microorganisms in the bloodstream of the prior host they were feeding on.

Sharing food, drinks, eating utensils, or shaking someone's hand will not transmit Lyme disease. But, a tainted blood transfusion could. You can't catch Lyme from your pet through natural contact, such as by grooming or caring for them. You can, however, get bitten by a tick that is on their body and that climbed onto you. Do not sleep with pets in your beds.

Lyme disease is a rampant epidemic in the 21st century. It's spreading in vast proportions. In 2013 the CDC upped the former 30,000 per year contagion estimations to over 300,000 people being infected yearly, but a mere $10 \%$ are being accurately diagnosed, due to limited physician and public symptom awareness, and very inaccurate lab testing, originated in the early 1980's and not updated. Lyme in the USA is spreading 4times faster than HIV. It is akin to the explosion of the polio epidemic 65years ago, with a large focal point in summer season. All of us are vulnerable; children playing outside or on sports fields, anyone of golf courses, gardening, hiking, fishing, camping or equestrians.

Low lying woodland areas, beach grass, and open meadows abutting forests are all favorable environments for ticks to inhabit.
Ticks typically find a small animal, such as a rabbit, squirrel, raccoon, dog, or deer to live on as their host. They rest on leaves and grasses, climbing aboard a warm-blooded animal (including humans) as they scrape by. The tick crawls on the body, looking for soft delicate skin as its entry point. They often gravitate to the nape of the neck, the armpits, and groin and attach to the host, often lodging themselves for hours or days, as they engorge on blood. A tick must lodge itself, not just crawl on you, in order to transfer the bacteria. Much speculation abounds, suggesting the tick must be embedded for over 24 hours for the blood transfer. Data does not support this well intended reassuring conclusion.

Spring and autumn a particularly elevated time for tick numbers as this is when their eggs cycles hatch out. After a rain, their presence can be heavier, too. Always check your limbs and clothing when coming back from a walk, gardening, fishing, farming. Do daily tick checks: scanning your body; feeling behind ears, in the hair, and along our limbs, at bedtime. It's wise to check your family pets regularly, too. Spray with insect repellent before going into the woods, meadows, and beach dunes is strongly recommended as a deterrent. DEET does not always deter ticks. A permethrin (derived from chrysanthemum) repellent, is effective, as are natural offerings made from lemon balm, eucalyptus and rose geranium. Long sleeves, pant legs tucked into socks help. Please check your young children daily and teach the older ones to examine themselves nightly, just as you routinely brush your teeth.

Consider having your pets vaccinated against Lyme disease. You can also use tick/flea baths and repellents on your pets. Pest companies can spray your yards, ball fields and public picnic areas with permethrin or cedar oil. The black-legged tick, American dog tick, Lone Star tick, Western black-legged tick, and Pacific coast tick, all Lyme carriers. The bird fly-ways have transported infected ticks everywhere in North America, Europe, Asia and Australia.

The most telltale sign of a new Lyme infection is the "bull's-eye" rash from the bite of the tick. A red dot in the center, with an outer red circle is the classic early warning sign. This target-shaped rash may be as small as a dime or as large as a softball. Sometimes it's a pale pink 
color, other times it's a vermillion red, even inflamed to a swollen, sore, black and blue degree.

Many people never notice such a bite. Less than 50 percent show the target rash. A small bull's-eye could also be in a not so visible place, such as your back or scalp. If you think a red welt or bite could be a common spider bite - also sometimes a red dot encircled with a red ring -please get it examined. With Lyme disease escalating so rapidly and its consequences being so dire, it's not worth brushing off such a bite as insignificant. Men, please note: You're not being a 'wimp' if you have an insect bite examined!

Other early symptoms of a Lyme infection include any combination of an aching, flu-like feeling in the limbs, back and neck, mild chills, nausea, headache, a slight fever. Occasionally some individuals will exhibit a frank swelling or pronounced pain in their joints (the knee being the most common). Dizziness, sore throat, noted fatigue, heart palpitations, and swollen glands may all manifest. Depression is not atypical.

Symptomology tends to linger beyond a few days. What later sets in is a heavy malaise and pronounced fatigue, often accompanied by a "cottony" head sensation, and mental dullness. Some cases occasionally bloom into a pronounced influenza state, whereby one discounts the notion of Lyme at all. Individuals with very strong immune systems may get these flu-like symptoms for only a few days, to relapse again weeks or even months later.

Please note that children may not get all the mentioned symptoms. Instead they most commonly manifest achiness and headaches, and maybe fatigue. Because children so frequently experience mild fevers, sore throats, and tiredness associated with a touch of some passing viral infection going through their school or daycare, we tend to just ride out their discomforts for a few days or a week, assuming it will all pass, as their active immune system capably arrests the microbe invasion.

If your child or teen complains of headaches, is unfocused in school, is showing anxiety or restlessness, has a knee or other joint that hurts, or just doesn't seem to have his or her usual energy, a few weeks after a seemingly mild cold of flu-like episode, have them tested for Lyme disease. Children, teens, and very healthy adults often are misdiagnosed regarding early Lyme infections, especially if they didn't exhibit the bull's-eye rash. The result is early, undiagnosed signs of Lyme, then a quieter waiting period, with malingering symptoms sometimes resurfacing weeks or months later, whereby no one, doctor or patient, makes the correlation to the initial infection. This scenario is where most chronic Lyme cases have gone awry. They were not diagnosed up front, setting up the individual for repeated suspicious outbreaks, eventually morphing into the mysterious and vague status of persistent Lyme disease, sometimes appearing like auto-immune diseases, often with serious neurologic or cardiac consequences.

Many physicians aren't willing to acknowledge the concept of persistent Lyme disease, as the IDSA (Infectious Disease Society of America) still labels Lyme disease as only an acute illness of a limited duration, from early definition in 1975. Yet, chronic forms of Lyme disease are very evasive, defying a clear black and white set of diagnostic keynotes. It may attack one or more primary system of the body, consequently creating a broad variety of symptoms variant from case to case.

As the spirochete augers from bloodstream to connective tissue, organs, glands and spinal fluid assorted symptoms spark: Parkinson's, various types of palsy, MS, Lupus, fibromyalgia, manic-depressive disorder, obsessive-compulsive disorder, and hallucinations, and even heart troubles, such as peri-carditis or valve problems occur. A friend's child had ocular Lyme and was blind until treated. Many people are hospitalized from Lyme disease, especially when the immune system becomes so overburdened that secondary infections, like pneumonia hit, or the inflammation is so severe that paralysis manifests. Chronic fatigue Syndrome, rheumatoid arthritis, persistent migraines and lupus are common signs of a advanced Lyme. Lyme takes the physical, mental and emotional stuffing out of so many people and their caregivers.

This rampaging illness of the 21 st century has showed us its very ugly face. Millions worldwide are suffering at so many levels because of it. A clinical diagnosis is made by a symptom picture, backed up with new state-of-the art laboratory testing that identifies DNA fragments of Lyme bacteria and specific reactive proteins more closely, than the traditional ELISA test or CDC criterion Western Blot antibody test. Local commercial lab testing is very faulty. IgeneX labs, Neuroscience iSpot test and Galaxy Labs are the current state of the art testing labs to insist your physician use. Look up their websites.

This hidden epidemic is finally gaining recognition with celebrities like Avril LaVigne, Debbie Gibson, Yolanda Foster stepping forth as afflicted. Visit ILDAS.org for physician training and key symptoms or LymeDisease.org for location of support groups.

\section{Biography}

Katina I. Makris, CCH, CIH is host of "Lyme Light Radio", author of "Out of The Woods, Healing Lyme Disease, Body, Mind \& Spirit" and "The Autoimmune Illness \& Lyme Disease Recovery Guide, Mending Body, Mind \& Spirit" published by Helios Press. Keynote Speaker of The Ticked Off Music Series, Lyme disease educator, Classical Homeopath and Certified Spiritual Healer.

Finalist US Book News Award in Alternative Health Winner "Top 50 Authors You Should Be Reading. The Author's Show. Katina is a graduate of Duke University and The Still point School of Integrative Life Healing. She sat on the board of the Council for Homeopathic Certification for 7 years.

\section{Acknowledgments}

None.

\section{Conflicts of interest}

Author declares there are no conflicts of interest.

\section{Funding}

None. 